\title{
Ultrafast signal processing in quantum dot amplifiers through effective spectral holeburning
}

Berg, Tommy Winther; Mørk, Jesper; Uskov, A. V.

Published in:

Summaries of Papers Presented at the Lasers and Electro-Optics, 2002. CLEO '02. Technical Digest.

Link to article, DOI:

10.1109/CLEO.2002.1034452

Publication date:

2002

Document Version

Publisher's PDF, also known as Version of record

Link back to DTU Orbit

Citation (APA):

Berg, T. W., Mørk, J., \& Uskov, A. V. (2002). Ultrafast signal processing in quantum dot amplifiers through effective spectral holeburning. In Summaries of Papers Presented at the Lasers and Electro-Optics, 2002. CLEO '02. Technical Digest. (Vol. 1). IEEE. https://doi.org/10.1109/CLEO.2002.1034452

\section{General rights}

Copyright and moral rights for the publications made accessible in the public portal are retained by the authors and/or other copyright owners and it is a condition of accessing publications that users recognise and abide by the legal requirements associated with these rights.

- Users may download and print one copy of any publication from the public portal for the purpose of private study or research.

- You may not further distribute the material or use it for any profit-making activity or commercial gain

- You may freely distribute the URL identifying the publication in the public portal 
CFH7

11:45 am

Ultrafast Signal Processing in Quantum Dot Amplifiers Through Effective Spectral Holeburning

T.W. Berg, J. Mork, Research Center COM, Technical University of Denmark, Build. 345V, DK-2800 Kgs. Lyngby, Denmark, Email: twb@com.dtu.dk

A.V. Uskov, Lebedev Physical Institute, Leninsky pr. 53, 117924, Moscow, Russia

Significant progress has been obtained on Quantum dot (QD) lasers, ${ }^{1}$ but the possible advantages of $Q D$ amplifiers are not yet clear. We show here that a relatively slow coupling between the optically active QD carrier states and the surrounding carrier reservoir can lead to efficient gain modulation suitable for ultrafast signal processing. The basis of this property is that the process of Spectral Hole Burning (SHB) can become very effective.

We consider a traveling wave optical amplifier consisting of the dot states, which interact with the optical signal (no inhomogeneous broadening included), and the Wetting Layer (WL), where current is injected. Time evolution is described by two coupled rate equations. Carrier capture from WL to dots is characterized by the capture time $\tau_{0}$.

Fig. 1 shows the induced gain change in the QD's under injection of a modulated optical sig

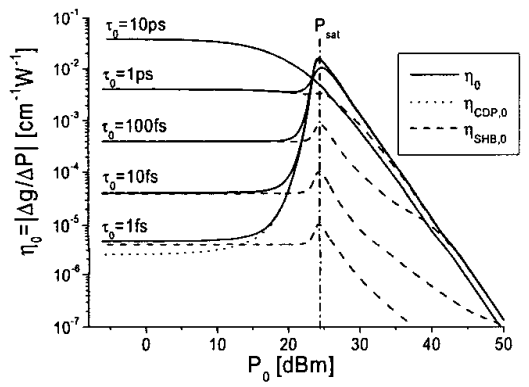

CFH7 Fig. 1. The low frequency response (induced small signal gain change), $\eta_{\theta}$, as function of DC optical power for five different values of the carrier capture time $\tau_{0}$. The CDP contribution is shown with a dotted line (not changing with $\tau_{0}$ ) and the SHB contribution with dashed lines.

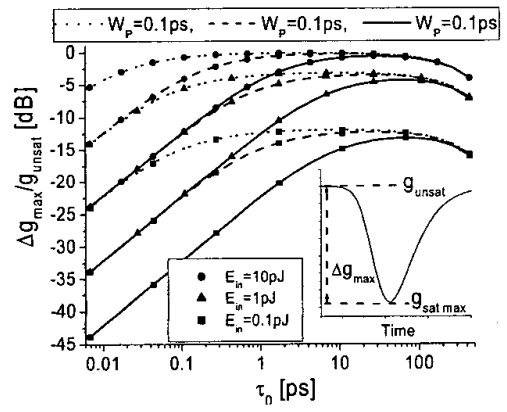

CFH7 Fig. 2. Maximum gain change induced by a single pulse for three different pulsewidths, $W_{p}$, and three different pulse energies, $E_{i n}$. The insert illustrates the definition of $\Delta g_{\max }$. nal with DC component, $P_{0}$, and modulation amplitude, $\triangle P$. The response is seen to consist of two parts: a SHB component, dominating for small $P_{p}$, where the amplifier is strongly inverted, and a Carrier Density Pulsation (CDP) component, which dominates when the amplifier starts to saturate. The longer the capture time, the stronger the induced gain modulation, i.e. a device with a relatively slow capture mechanism driven in the strong inversion regime can exhibit efficient gain modulation independent of average power (no pattern dependence). This is in contrast to ordinary signal processing, in bulk or QW devices, which is based on the CDP effect, i.e. inherently limited by the spontaneous lifetime and if driven above this limit, strongly pattern dependent. ${ }^{2}$

Fig. 2 shows, as function of $\tau_{0}$, the maximum gain change, $\Delta g_{\max }$ induced in the device when a single pulse is injected. For $\tau_{0}<W_{p} \Delta g_{\max }$ is determined by the pulse peak and for $\tau_{0}>W_{p}$ it is determined by $E_{i n}{ }^{3}$ For given $W_{P}$ and $E_{i n}, \Delta g_{\max }$ increases linearly with $\tau_{0}$ and, therefore, a device with $\tau_{0} \approx W_{P}$ is to be preferred in this context. Capture times on the order of 5-10 picoseconds have been reported for InAs/GaAs dots at room temperature, ${ }^{4}$ indicating the potential as good candidates for efficient gain modulation at high speeds. Since $\tau_{\theta}$ determines the speed limitation it should not be longer than $\approx 1 / 10$ of the available timeslot in order to avoid strong patterning effects.

We now consider the case where a $\mathrm{QD}$ amplifier is used for modulating (switching) a CW signal with a $100 \mathrm{Gbit} / \mathrm{s}$ optical data signal through the cross-gain effect. ${ }^{2}$ The results (Fig. 3) show some pattern dependence after $\approx 10$ pulses due to depletion of the WL. ${ }^{5}$ This is diminished if injecting weaker pulses, but at the cost of a degradation of the extinction ratio. The system is clearly able to operate efficiently at this speed. Since the principle of operation described here is based on the SHB effect, the maximum detuning between data signal and converted signal is limited by the homogeneous linewidth, suggesting that this technique may be viable for signal processing, but not for wavelength conversion. The broad inhomogeneously broadened spectrum of self-assembled QD's may allow for processing of several signals simultaneously.

1. R.L. Sellin, Ch. Ribbat, M. Grundmann, N.N. Ledentsov, D. Bimberg, "Close-to-ideal device characteristics of high-power InGaAs/

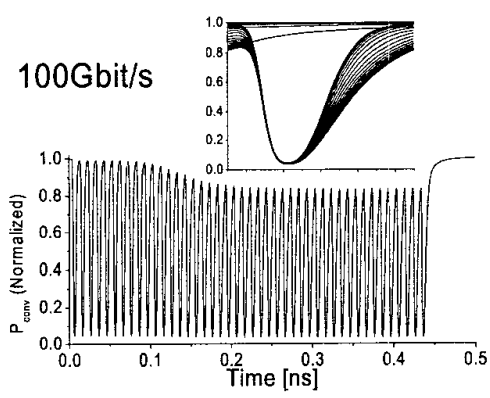

CFH7 Fig. 3. Time evolution of a converted signal and the corresponding calculated eye (insert) for a QD amplifier with $\tau_{0}=1 p s$ and $20 \mathrm{~dB}$ gain ( $4.5 \mathrm{~mm}$ long device). The injected signal is a worst-case signal consisting of a long string of ' 1 's $\left(W_{p}=1 p s\right)$ followed by a long string of ' 0 's.
GaAs quantum dot lasers", Appl. Phys. Lett., 78, 1207-1209 (2001).

2. T. Durhuus, B. Mikkelsen, C. Joergensen, S.L. Danielsen, K.E. Stubkjaer, "All-Optical Wavelength Conversion by Semiconductor Optical Amplifiers", J. Lightwave Technol., 14, 942-954 (1996).

3. A.V. Uskov, J. Mørk, J. Mark, "Theory of short-pulse gain saturation in semiconductor laser amplifiers", IEEE Photon Technol. Lett., 4, 443-446 (1992).

4. J. Feldmann, S.T. Cundiff, M. Arzberger, G. Böhm, G. Abstreiter, "Carrier capture into InAs/GaAs quantum dots via multiple optical phonon emission", J. Appl. Phys., 89, 1180-1183 (2001).

5. T.W. Berg, S. Bischoff, I. Magnusdottir, J. Mørk, "Ultrafast Gain Recovery and Modulation Limitations in Self-Assembled Quantum-Dot Devices", IEEE Photon Technol. Lett., 13, 541-543 (2001).

CFI

10:15 am-12:00 pm Room: 104A

Pulsed Terahertz-Beam Applications

$X i-C h e n g$ Zhang, Rensselaer Polytechnic Inst., USA, Presider

CFI1 Invited

10:15 am

Terahertz Excitation Dynamics in Semiconductor Heterostructures

Roland Kersting, Department of Physics, Rensselaer Polytechnic Institute, Troy, NY

Intersubband transitions in semiconductor heterostructures constitute an ideal multi-level system for quantum optical applications. Compared to atomic or molecular systems, the resonances of intersubband structures can be tailored and offer direct electronic control. Moreover, giant transition dipole moments can be designed in the farinfrared, which allows to enter the nonlinear excitation regime at extremely low light intensities. Recently, these properties have stimulated the de velopment of new concepts for photonic signal processing at terahert $z$ frequencies.

The advent of terahertz time-domain spectroscopy (THz-TDS) gave research on intersubband dynamics a new dimension of time resolution. THz-TDS allows the measurement of a transient polarization with subcycle time resolution thus providing full information on the intersubband response to ultrafast and nonadiabatic excitation pulses.

We have investigated the dynamics of intersubband excitations in modulation $\mathrm{n}$-doped $\mathrm{Al}$ $\mathrm{GaAs} / \mathrm{GaAs}$ heterostructures. Their parabolically shaped conduction band leads to equally spaced intersubband transitions, which have typical resonance frequencies between $1.5 \mathrm{THz}$ and 3.0 THz. The quantum wells are modulation $\mathrm{n}$-doped at about $10^{12} \mathrm{~cm}^{-2}$ and have electron mobilities of about $80,000 \mathrm{~cm}^{2} / \mathrm{Vs}$, which correspond to dephasing times of about 2 ps. The electron density in the quantum wells can be controlled electronically by the Schottky grating contacts fabricated on top of the structures. 